\title{
Germanica
}

GERMANICA $\quad 12 \mid 1993$

Le Modernisme dans les littératures scandinaves

\section{La percée du modernisme dans la littérature nordique}

Durchbruch des Modernismus in der nordischen Literatur

\section{Torben Brostrøm}

Traducteur : Ingrid Renard

\section{(2) OpenEdition \\ Journals}

\section{Édition électronique}

URL : http://journals.openedition.org/germanica/430

DOI : 10.4000/germanica.430

ISSN : 2107-0784

\section{Éditeur}

Université de Lille

\section{Édition imprimée}

Date de publication : 1 juin 1993

Pagination : 13-33

ISSN : 0984-2632

\section{Référence électronique}

Torben Brostrøm, «La percée du modernisme dans la littérature nordique », Germanica [En ligne], 12 | 1993, mis en ligne le 11 avril 2013, consulté le 06 octobre 2020. URL : http://journals.openedition.org/ germanica/430; DOI : https://doi.org/10.4000/germanica.430

Ce document a été généré automatiquement le 6 octobre 2020.

(c) Tous droits réservés 


\title{
La percée du modernisme dans la littérature nordique
}

\author{
Durchbruch des Modernismus in der nordischen Literatur
}

\section{Torben Brostrøm}

Traduction : Ingrid Renard

1 Le modernisme est une pieuvre, un monstre aux tentacules multiples. C'est un mollusque sans forme définie dont il existe de nombreuses variétés. Il se sert de ses multiples tentacules aussi bien pour marcher que pour capturer sa proie. Il peut se mouvoir avec une grande puissance, ou se laisser porter sans effort. La sécrétion noire de sa glande, son "encre", sert à brouiller les eaux de manière à surprendre ses adversaires ou à échapper à un ennemi.

2 Si je compare ce mouvement dans le domaine des arts à une pieuvre, c'est parce que le modernisme est un animal étrange, extrêmement vieux et pourtant toujours en vie, suscitant l'attention, suscitant chez beaucoup même l'effroi, insaisissable, en perpétuel mouvement et s'étendant dans toutes les directions. Certes, on peut le définir et déterminer son espèce. Mais si l'on parle du modernisme, il faut ne faut pas oublier qu'il s'exprime de multiples façons qui prennent toujours leur point de départ dans un potentiel de protestation, mais sous une forme ludique. À la manière dont Gunnar Ekelöf décrit ses animaux totémiques dans En natt i Otocac (Une nuit à Otocac) (1961) :

$\mathrm{Au}$ fond de l'eau se trouve la pieuvre à huit bras et au dessus d'elle, six ou sept sepias qui dansent comme des oiseaux sous-marins ou des papillons :

« Je sais bien à quoi ils jouent

ils jouent au fleuve, fleuve des abîmes, contre-courant glacé,

qui les force à danser sur place

au-dessus des abîmes qui guettent ${ }^{1}$ ".

3 À tout moment, dans le modernisme, on a le sentiment d'un abîme qui guette, d'une absence peut-être, d'un vide, d'une perte de sens; alors qu'en surface une danse endiablée aux innombrables variations traduit l'ivresse d'être délivré d'un vieux poids mort. 

de l'expression artistique : peinture, sculpture, musique, poésie, architecture, théâtre, scénographie, cinéma, danse, mode, gastronomie, philosophie. Dans sa forme la plus dense, cette révolution eut lieu durant la deuxième décennie du vingtième siècle, plus précisément, entre 1909 et 1924. Si l'on pense en termes de littérature, cela va du manifeste futuriste au manifeste surréaliste. Mais, en fait, ce mouvement n'a jamais cessé d'exister, bien qu'aujourd'hui on parle plutôt de post-modernisme. Dans cette grande rupture avec les normes en cours, les concepts communément admis sont à la fois de nature positive et négative : une révolte contre le naturalisme et le romantisme national et une profession de foi envers la nouvelle époque, envers l'internationale de l'art. La notion d'avant-garde était ainsi lancée, les concepts de formes et les mots étaient entièrement libérés. Parole in libertà. L'héritage culturel fut jugé, l'harmonie rompue. Pour les remplacer on rêva d'un monde où « tous les préjugés harmonieux font défaut, / où les couleurs éclatent, où les formes sont dispersées / et où la beauté naît de conflits éclatants »- pour citer le poète Tom Kristensen.

Si l'on cherche à savoir en quoi le contexte social a provoqué la rupture à ce moment précis, on trouvera facilement une série de causes concordantes dans le développement économique des pays industrialisés, la croissance rapide de la nouvelle technologie et du capital, ainsi que la naissance d'une pensée politique révolutionnaire hostile à la bourgeoisie. Il faut y ajouter le développement de la culture de masse, en raison notamment de l'influence américaine sur l'Europe de l'Ouest.

7 Plusieurs de ces tendances renvoient au passé. Chaque fois qu'il s'agit de dater un phénomène, on est obligé de relativiser son origine. À chaque supposition, sa présupposition. Dans l'histoire de la littérature, le prélude au modernisme est décrit de manière très diverse. Certains le trouvent déjà à la fin du XVIII ${ }^{e}$ siècle avec la consécration de l'artiste en tant qu'individu et l'apparition de l'institution littéraire. D'autres trouvent des traces dans la poésie baroque et disent: regardez comme c'est moderne ! D'autres encore voient le poète romantique trahir son déchirement intérieur dans l'évocation de belles images, comme c'est le cas chez Stagnelieus ou Schack Staffeldt.

En ce qui concerne l'étude de la poésie, il est devenu courant de se référer à deux voies d'accès, l'une passe par le Nouveau monde, l'autre par l'ancien. Elles ont été ouvertes à peu près au même moment par deux recueils de poèmes : Leaves of grass (1855) de Walt Whitman et Les Fleurs du Mal (1857) de Charles Baudelaire. Il s'agit d'une part d'un hommage extatique à la période moderne et à son dynamisme, et d'autre part d'un attachement conflictuel à un vieux monde qui s'effondre. Ce sont deux marginaux qui vivent, l'un dans la République démocratique américaine, l'autre au milieu de la bourgeoisie française du Second Empire. Whitman concilia toutes les contradictions de l'existence en s'élevant au dessus d'elles. Baudelaire analysa le schisme et la décadence dans sa recherche même de la nouveauté. Trouver la nouveauté dans ce qui nous échappe, voilà précisément une morale moderniste.

9 Le modernisme est inséparable de tout changement de civilisation. Cela représente un processus de longue durée, qui se déroule dans le conflit incessant entre, d'une part de nouvelles expériences, de nouveaux vécus, et d'autre part une forme attachée à la

Germanica, 12 | 1993 
tradition. On peut dire qu'il y a deux directions : l'une qui mène à des heurts et des collisions, l'autre qui s'échappe vers les cieux. Une communication qui passe par un sac de nœuds ou alors un imaginaire débridé.

Lorsque l'on dit que le modernisme est anti-naturaliste, il s'agit d'une vérité qu'il faut nuancer. C'est peut-être vrai en ce qui concerne la peinture. En littérature, où le naturalisme imitait la réalité, la nouvelle période fit une irruption si brutale que cela pouvait paraître étrange et choquant. Le langage se mit à former d'étranges guirlandes imaginaires qui ne pouvaient être rattachées à aucune réalité. Un poète comme J.-P. Jacobsen, qui était un naturaliste déclaré, créa un nouveau langage poétique avec ses Arabesques. En tant qu'athée, il devait avouer que le monde lui semblait vide. Il n'était cependant pas capable de supprimer sur commande son ancrage religieux, bien qu'au fond de lui-même il le rejetât. Le voilà qui se retrouve avec son idéalisme vide, une transcendance vaine qui envahit le langage émotionnel avec des métaphores singulières. L'image poétique se libère et brise les structures fixes de la pensée. La philosophie naturaliste, positiviste, a tendance à démolir les entités, à atomiser la connaissance. Le pessimisme et le nihilisme en furent les conséquences. Ce fut aussi le début d'une division, d'un déchirement de l'âme moderne qui se poursuit dans certaines branches du modernisme. D'une certaine manière l'art et l'esthétique devenaient un ersatz de la religion, le sentiment religieux se réfugiant dans la forme artistique. Rainer Maria Rilke apprit le danois afin de pouvoir lire le roman de J.P. Jacobsen sur le libre penseur Niels Lyne et traduire ses " Arabesques».

11 Le mode de pensée scientifique du naturalisme, ancré dans le monde physique et biologique, est, lui aussi, créateur d'un langage qui préfigure le modernisme. August Strindberg évoque dans ses Dikter (Poèmes) (1883) les impressions issues de son premier voyage à l'étranger. Il s'agit d'un mélange, tout à fait nouveau, de poésie et de diction en prose qui allait libérer la forme figée. Ce qui devait caractériser le modernisme était en germe depuis longtemps.

Le symbolisme ou néo-romantisme est tout aussi ambigu que le naturalisme en tant qu'idéologie moderniste. Le terme de symbolisme renvoie à une conception globalisante. Il est possible de voir des correspondances entre le monde matériel et le monde spirituel et de concevoir l'existence en tant qu'idée, physique et métaphysique se complétant mutuellement. Mais le symbolisme est un idéalisme désespéré, une ultime tentative de surmonter la scission entre la plénitude et le vide, le langage y apparaissant comme une bouée de sauvetage. Grâce à ses manipulations de la langue et à l'imagination, le symbolisme parvint à trouver la solution du déchirement de la conscience moderne, la poésie elle-même, l'expression linguistique, l'image poétique, deviennent source de salut, construction héroïque d'une passerelle comme chez Sophus Claussen, Vilhelm Ekelund, Olaf Bull, chez Eino Leino et Einar Benediktsson. Mais le néant est à l'affût, comme le dit Sigbjørn Obstfelder dans son celebre poème : Jeg ser (Je vois) (1893). Le poète a souvent été représenté, ou s'est présenté lui-même, comme un visionnaire, une sibylle, un sage, ou, selon Rimbaud, comme un "voyant» qui fouette son imagination. Dans le naturalisme, c'est un "voyeur", celui qui surprend les singularités humaines. Mais chez Obstfelder il y a comme un constat surprenant, le symboliste voit soudain tomber les décors :

Je vois le ciel blanc je vois les nuages bleu gris, je vois le soleil sanglant. 
Voilà donc le monde.

Voilà donc le foyer des planètes.

Une goutte de pluie! expressionnistes qu'une dizaine d'années plus tard. Nous trouvons déjà tout l'attirail du modernisme du XXe siècle dans les lignes suivantes :

Silence ! regarde comme la machine

cette chose massive, boue calmement

et s'enveloppe de fumée, elle est patiente.

Allume ta pipe à jeun,

maudit Dieu et avale ta douleur ${ }^{3}$.

$17 \mathrm{~J}$.-V. Jensen les a écrites alors qu'il se trouvait dans la gare de Memphis, Tennesee, aux U.S.A., en train d'observer le monde nouveau: la machine sûre de sa victoire, tranquille, sachant qu'elle va conquérir le monde. Plutôt que de s'abandonner aux lamentations et aux appréhensions, il prend une décision; il allume sa pipe et s'identifie à la machine qui fume. Il maudit Dieu, et abjure ainsi son passé métaphysique. Il ne peut pas faire autrement malgré la gravité de son acte. Il transforme la nécessité en vertu.

18 Nous ne sommes pas très loin de la morale moderniste. Faire de la nécessité une vertu. Trouver un enthousiasme dans la perte. L'effondrement de l'ancien monde était un fait. 
La mort de Dieu avait été proclamée par Nietzsche, la conception symboliste paraissait de plus en plus forcée, le monde devenait vide et étranger. Il y a dans le modernisme de nombreuses descriptions de cet effondrement, aussi bien en poésie qu'en prose. Il suffit de penser à Rainer Maria Rilke et à Franz Kafka qui ont chacun leur propre version du processus.

Mais le grand souffle de renouvellement qui traversa l'art européen à partir de 1909 était avant tout une vague d'enthousiasme, une ivresse devant l'infini des nouvelles possibilités d'expression. De façon très générale; c'était là le passage d'un langage symbolique contraignant à un libre langage des signes.

Une brève esquisse de la situation des «ismes » en Europe : à Paris, le cubisme naquit dans le cercle de peintres et d'écrivains autour de Picasso. C'était un milieu où fusaient les idées, où cohabitaient toutes les branches de l'art. On constate souvent que la peinture est à la tête des renouvellements modernistes. C'est un outil plus sensible encore que la poésie. C'est aussi à Paris que l'italien Marinetti publia son manifeste futuriste qui, dans ses onze points, exalte la beauté de la vitesse, l'agressivité de la littérature, la puissance purificatrice de la guerre et recommande la destruction des musées, des bibliothèques, des académies, de la morale et des mouvements féministes. Le courage, l'audace et la révolte de la poésie doivent jouer un rôle décisif dans le développement de la nouvelle époque et de sa technique.

On peut s'étonner de la force avec laquelle s'imposèrent ces vulgarités, qui allaient bientôt épouser la cause du fascisme italien. Mais la dynamique du futurisme n'était pas liée à ce dernier, ce que montre par exemple sa version russe, avec le manifeste de 1912: Une gifle au goût ordinaire, de Burljuk, Chlebnikov, Kručonih et Majakovskij : " Nous seuls, nous représentons le visage de notre temps - À travers nous, le temps fait sonner son cor dans l'art des mots".

Le futurisme russe pouvait participer à la remise en question de la culture bourgeoise dans le cadre de la révolution à venir. Mussolini et Trotski avaient une idée très différente de la manière dont ils pouvaient utiliser le nouvel art des mots. Ils avaient en commun leur intérêt pour le dépassement du moi quotidien et de son univers limité, afin que le poète et les mots entrent dans une relation active avec le réel. Se trouver sur le « rocher linguistique du nous » comme il est dit dans le manifeste des quatre.

L'expressionnisme était un vaste mouvement, qui s'était développé surtout en Allemagne avec les revues Der Sturm et Aktion, plus engagée politiquement. L'idéologie de l'expressionnisme est plus souple que celle du futurisme. Elle construit son utopie sur l'homme, ou l'homme nouveau - ou l'être $n u$ comme on disait. Elle a un caractère humaniste, bien que l'on puisse exprimer des doutes à cause de l'accent mis sur l'irrationalité, l'instinct, l'intuition et les pulsions. Ce sont cependant des éléments qui entrent en compte dans toute création artistique, et l'esthétique expressionniste, avec sa proclamation de la souveraineté de l'expression, eut une influence très vaste, indépendamment de toute idéologie. L'ambiguïté de ses tendances stylistiques se révéla cependant rapidement lorsqu'au premier enthousiasme dû à la libération se mêla le désespoir causé par l'effondrement des valeurs, que la guerre mondiale devait confirmer. Le cri expressionniste est sans doute devenu le signe même de ce mouvement. 


\section{(1)}

erture à l'inconscient. L'outil en fut baptisé « écriture automatique ». On échappait ainsi au contrôle de la raison pour accéder à d'autres causalités. Il s'agissait de parvenir à de nouvelles formes d'expression pour les choses cachées, des contacts secrets de la vie psychique et des liens profonds entre les hasards extérieurs de l'existence. Il est tout à fait caractéristique que le dernier des grands "ismes » modernistes fut un mouvement qui chercha à établir des liens cohérents, à construire des valeurs, en théorie et dans la pratique; d'une certaine façon ce fut un nouveau romantisme, un nouveau symbolisme, un ersatz de religion. La pratique poétique se révéla d'une extrême complexité avec le libre flot des images, les combinaisons de rêves. Elle s'oppose en cela à la dernière, mais non pas la plus récente, des esthétiques fondamentales, l'imagisme, qui considérait que le poète devait purifier la langue et la mener à une simplicité et à une précision aussi grande que possible grâce à un nombre limité d'images significatives : les idéogrammes de l'âme. L'imagisme peut-être daté de 1909, l'année même de l'introduction du modernisme, l'année où Ezra Pound arriva en Angleterre et tenta d'en finir avec la poésie traditionnelle. Peu après il s'installa à Paris, comme tant d'autres Américains et Anglais.

26 Le modernisme d'Europe centrale expérimenta en quinze ans, à en juger par les manifestes, tout le champ des expériences et des possibilités d'expression de l'homme moderne, ainsi que de sa conception chaotique du monde. Dans les années qui suivirent, on en récolta les fruits dans toute une série de domaines, par la mise en œuvre de toutes sortes d'expériences pratiques, dans différents pays, dont les pays nordiques. Certains ont affirmé que depuis, on n'en a plus connu que des variations et des mélanges, actualisés dans certains contextes de lieu et de temps.

L'étude du modernisme nordique en littérature est, à peu de choses près, un exercice de littérature comparée. On met bout à bout ce qui s'est passé dans les différents pays, en étudiant le comment et le pourquoi. Dans un certain nombre de cas, on peut parler d'influences directes et d'emprunts. On pourrait également, en employant un vocabulaire plus moderne, parler d'intertextualité et d'expériences communes. Le poète finlandais Elmer Diktonius disait : «Le modernisme était dans l'air, ses cris et ses appels étaient nés pendant la guerre mondiale, il s'était nourri des bouleversements, l'angoisse et l'attente de l'époque l'avaient fait pénétrer au plus profond de nousmêmes ». 

l'éclat particulier qui émane de ses poèmes, et cela dès ses Dikter de 1916. Mais c'est avec Septemberlyran (La Lyre de septembre) de 1918 et les recueils suivants, Rosenaltaret (L'autel des roses) et I Framtidens skugga (À l'ombre de l'avenir) (1919 et 1920) qu'eut lieu la formidable percée d'un nouveau sentiment du moi, témoignant de la grande conscience que la poétesse avait d'elle-même : «Mon assurance vient de ce que j'ai découvert mes dimensions » écrit-elle. Son expressionnisme euphorique fut stimulé par sa rencontre avec la pensée de Nietzsche et par le caractère exceptionnel de son internationalisme : elle avait fréquenté une école de langue allemande à Saint-Petersbourg, vécu à Raivola sur la pointe de la Carélie, fait de longs séjours en sanatorium en Suisse etc. Elle était de retour à Saint-Petersbourg peu après la Révolution d'Octobre. Tenant une place à la fois marginale et centrale, elle était l'incarnation vivante de l'Europe malade et de cette « culture de sanatorium » décrite par Thomas Mann dans La Montagne magique. Grâce à sa poésie, elle en fit un monde enchanté. Elle transforma la nécessité en vertu. Elle fut la prêtresse et la sibylle du mouvement moderniste en Finlande, qui débuta en 1922 avec la revue bilingue Ultra, dirigée par Hagar Olsson et Elmer Diktonius, et qui s'épanouit à travers les nombreuses ramifications de l'expressionnisme et un dadaisme révolutionnaire, avec des personnalités aussi diverses que Gunnar Björling, Rabbe Enkell, Henry Parland. Diktonius déclara dans son article sur l'expressionnisme (Ultra 1922) : « Notre être intime est projeté hors de nous-mêmes, en débris explosifs, livré plus nu que ne le fut jamais le corps humain lui-même dans l'art antique ». Le sentiment du moi est universel et englobe tout. L'invitation lancée aux souscripteurs de la revue le précisait bien : « Nous comprenons tout le monde, les sauvages, les païens et les chrétiens, les Chinois, les Allemands, les Anglais... ».

31 Bønnelycke, puis Tom Kristensen, mais en finnois. De toute évidence il y a eu des contact avec le Danemark, avec Frederik Nygaard, qui débuta en 1915 et dont on connait les trois recueils de poésie écrits dans une forme libre, qui retrace ses impressions de voyage dans toute l'Europe. C'est un pendant danois à Blaise Cendrars. Tous, en particulier Bønnelycke, écrivaient dans la revue d'avant-garde Klingen (1917-1929). Bien que ce fut avant tout l'organe de la nouvelle peinture, où le modernisme était introduit sous toutes ses formes, la littérature y fut tout de même 
incluse, en raison des expériences pluriartistiques déjà mentionnées. C'est elle qui publia les premiers poèmes visuels en danois, ainsi qu'un texte français recherchant des effets sonores. C'était de la poésie concrète, sur les traces d'Appolinaire. Bonnelycke avait, en outre, été fortement influencé par la revue expressionniste allemande Der Sturm. Déjà avant la guerre, il avait étudié le manifeste futuriste de Marinetti avec son ami Tom Kristensen. Ses textes dans Asfaltens sange (Chansons de l'asphalte) de 1918 sont tout à fait futuristes, un hommage au siècle et un reflet du manifeste. Il animait les soirées expressionnistes à Copenhague, les premières de leur genre dans le Nord.

Au Danemark, comme en Finlande, il y eut des scandales et des polémiques dans le sillage des modernistes. Concernant l'année 1922, la plus importante dans l'histoire du modernisme, l'année où parurent Hårda sånger (Chants cruels) de Diktonius, Fridas bok (Le Livre de Frida) de Birger Sjöberg, The waste land de T. S. Eliot et Ulysse de James Joyce, où Rilke était en train de relire les épreuves de ses Élégies de Duino, il faut encore mentionner deux poètes danois. C'est, en effet, l'année où furent publiés au Danemark les recueils de poèmes Blod (Sang) de Broby-Johansen et Parole de Harald Landt Momberg, tous les deux de caractère explicitement expressionniste ; les deux auteurs avaient d'ailleurs des rapports avec la revue Sturm, où ils étaient représentés par des poèmes. Alors que Momberg connut des débuts tranquilles, Broby-Johansen s'attira un procès à cause du caractère provocateur de ses textes, de leur radicalisme politique et social. Son esthétique expérimentale de la laideur était plus que ne pouvaient supporter les autorités et les moralistes bourgeois. Les deux livres furent promptement oubliés, et ne furent redécouverts qu'en 1960 après une nouvelle vague de modernisme. Il est étonnant de voir que ces poètes eux-mêmes ont tourné le dos aussi vite à l'expressionnisme. Broby-Johansen se consacra essentiellement à la politique et à la critique d'art, Bønnelycke devint moraliste et se convertit au christianisme, Tom Kristensen remit en question toute sa génération avec son article sur Den unge Lyrik og dens krise (La Jeune poésie et sa crise). Dans la nouvelle revue radicale, Kritisk Revy, de 1927, on mettait l'accent sur l'objectivité et le fonctionnalisme. Otto Gelsted publia dans la nouvelle revue moderniste Quosego une critique meurtrière de la poésie de Gunnar Björling. C'était pourtant le même Gelsted qui avait attiré l'attention sur les nouvelles tendances, avec son livre Expressionnisme (1919), et qui avait publié la même année, avec Johannes $\mathrm{V}$. Jensen, un choix important des poèmes de Walt Whitman.

Le livre de Gelsted sur l'expressionnisme s'exprimait avec prudence à propos des tendances extrémistes allemandes, il préférait le cubisme pur, celui qui transpose les structures d'une expérience sensorielle sur le plan intellectuel, plutôt que de s'adonner de manière sauvage aux sensations et à l'expression nue. Peut-être les idées structuralistes du cubisme eurent, elles aussi, une influence plus durable. Voici un exemple de ce que l'on pourrait appeler une perception cubiste. C'est Emil Bønnelycke qui écrit : "Tout résonne. Le vert et le bleu. Il y a un effet foudroyant dans la réaction de toutes les lignes, les unes par rapport aux autres. Les surfaces des champs, les triangles et les rectangles des maisons, les cubes et les coupoles des forts, des arbres et des nuages rythment leurs voûtes les unes au dessus des autres, dans un équilibre divin, oh! quel spectacle».

Il y a beaucoup de choses qui attestent le rôle de Copenhague pendant la Première guerre mondiale et durant la période qui suivit, en tant que lieu de rencontre entre le continent et le Nord, en particulier en ce qui concerne la peinture, mais aussi les autres 
arts. Parlant de peinture et de poésie, on est obligé de nuancer l'affirmation selon laquelle les Danois auraient été les premiers à adopter le modernisme. En 1913, le Suédois Pär Lagerkvist publia son pamphlet Art des mots, art des images, avec comme sous titre «De la décadence de la littérature moderne et de la vitalité de l'art moderne ». Il avait alors pris contact, à Paris, avec le cercle de Gertrude Stein, de Picasso et d'Appolinaire. Dans son essai il s'intéresse, comme plus tard Gelsted, surtout au cubisme, mais il ne néglige pas pour autant l'expressionnisme. Les deux « ismes » représentent respectivement le renouvellement intellectuel et émotionnel. En revanche, il garde très nettement ses distances avec le futurisme.

La chercheuse danoise Lise Loesch a souligné le caractère extrêmement masculin, viril de la révolte contre une époque décadente, son symbolisme et son romantisme national, qu'il s'agisse de Johannes V. Jensen, de Bønnelycke, Diktonius ou Lagerkvist. Quoi qu'il en soit, pour Lagerkvist l'esthétique du cubisme tend vers l'art pur, quel que soit le nom qu'on lui donne, abstraction pure où « concrétion » pure. Il est d'autant plus étonnant que les recueils de poèmes qui suivirent son essai : Motiv (1914) et en particulier Ångest (Angoisse) (1916), présentent un caractère si manifestement expressionniste. Autant son extase devant l'homme nouveau, l'être nu et les miracles des machines est puissante, autant son angoisse existentielle est désespérée. Les deux aspects que j'ai souligné en parlant dans mon introduction de Whitman et de Baudelaire, l'enthousiasme et la peur, s'avéreront inséparables désormais. Pär Lagerkvist exprime cela ainsi :

L'angoisse, l'angoisse est mon héritage

la blessure de ma gorge

le cri de mon cœur au monde.

ou bien encore :

Je me ris de toi, la bouche pleine

de sang et de hurlements rauques... pays natal. Le chercheur suédois Anders Palm, qui a contribué avec son article Modernisme ou dismorphisme?, à donner une image plus juste de la percée du modernisme nordique, souligne à propos de Lagerkvist qu'aussi bien le recueil de poèmes Ångest que son essai Modem teater (Théâtre moderne) furent écrits au Danemark ! Pendant plus de dix ans, il habita principalement le Danemark, étant marié à une Danoise et fréquentant le milieu culturel de Copenhague. Il est simplement surprenant de constater que les fenêtres du Danemark si ouvertes sur le continent dans ces premières années de révolution littéraire, se soient si rapidement refermées sur ellesmêmes.

En ce qui concerne la Suède, il est sans doute caractéristique que l'introduction du modernisme ait été le fait d'auteurs isolés. Il faut mentionner en premier lieu Birger Sjöberg, mais seulement à partir de 1926 avec Kriser og kransar (Crises et cercles) où s'opère une nouvelle et souveraine concentration des images. C'est à la fois le produit d'un provincialisme suédois profond et du journalisme international. Il fut le précurseur indispensable de la deuxième vague moderniste en Suède dans les années 1940, avec Mannen utan väg (L'Homme sans chemin) de Erik Lindegren qui représente un des sommets de l'errance existentielle autant que de la perfection esthétique. 

de Whitman, introduit par Artur Lundkvist. Nous sommes alors à la fin des années vingt. Après son début avec Glød (Ardeur) (1928) et Naket liv (Une vie nue), des recueils aux titres caractéristiques, il rédige l'anthologie de sa génération: Fem unge (Cinq jeunes) (1929). Lui et Harry Martinson, deux nomades cosmopolites, étaient considérés comme les représentants d'un vitalisme expansif, tandis que Gunnar Ekelöf avec son recueil Sent på jorden (Tard sur terre) (1932) cherchait son propre chemin vers les contrés intérieures de l'âme, une dimension bien plus tragique et problématique. C'est là encore une constellation qui reflète la dualité moderniste. Ils ont cependant en commun la longue tradition moderniste, issue de Rimbaud, de Mallarmé et en particulier des surréalistes, sans oublier les finno-suédois. Il faut ajouter que Lundkvist rencontra Whitman au Danemark et qu'il fut très marqué par le modernisme danois des années vingt. En 1925, durant un séjour à Copenhague, il se plongea dans l'œuvre de Bønnelycke, Tom Kristensen et Nygaard. C'était l'année où Tom Kristensen déclarait que la jeune poésie était en crise. On peut dire cependant que les Danois se retirèrent vite du jeu, laissant l'initiative à d'autres.

Jusqu'ici il était surtout question de la percée du modernisme en Finlande, au Danemark et en Suède, sans prendre en compte les autres pays et aires linguistiques Scandinaves. En effet, les mouvements, les regroupements littéraires n'y ont pas la même importance, il n'y a pas la même atmosphère d'avant-garde. En Norvège, cela s'explique sans doute par la récente conquête de l'indépendance nationale en 1905, après la suppression de l'Union avec la Suède. Cela n'invitait guère à s'intéresser à ce qui se passait hors des frontières. À quoi bon un futurisme dédaigneux des traditions ? Cela ne veut pas dire qu'il n'y ait pas eu une poésie moderne ou proche du modernisme en Norvège durant les premières décennies du siècle. On pourrait nommer Olaf Bull comme l'un de ces grands poètes originaux qui constituent à eux seuls tout un mouvement ; c'est un continuateur de cette poésie de la ville, dont Knut Hamsun donna un exemple si suggestif avec son livre La Faim. Olav Bull évita cependant les expérimentations formelles proprement dites. Parmi ceux qui se sont tenus éloignés de tout mouvement, on pourrait mentionner également Olav Aukrust, Olav Nygard, des poètes visionnaires écrivant en nynorsk, la nouvelle langue norvégienne. Ce n'est pas une poésie des métropoles, mais celle des campagnes et des montagnes.

La Norvège ne connaissait pas la même urbanisation que le Danemark et la Suède, ce qui explique que le modernisme n'y ait pas trouvé un terrain fertile à cette époque. À partir des années 30 on peut mentionner des poètes comme Emil Boysen, Claes Gill et Rolf Jacobsen. Chez ce dernier, l'univers de la grande ville fait peu à peu son apparition, grâce à des relations nouvelles et originales qu'il établit entre la technique et la nature. Il est étrange de constater qu'il y a des liens très peu marqués entre la poésie en finnosuédois et celle écrite en langue finnoise proprement dite. Elmer Diktonius était pourtant un écrivain bilingue, et durant une courte période il exista un groupe moderniste autour de Uuno Kailas. Celui-ci renonça cependant rapidement à la tendance futuriste pour se reconvertir à la tradition. Seul Katri Vala conserva son inspiration, celle qui lui venait de Edith Södergran. 

Thordarsson qui était essentiellement un romancier. Halldor Laxness, un expert en tout, s'attaqua également, une seule et unique fois, au genre poétique, en 1930, le faisant éclater pour créer une forme humoristique du surréalisme, un jeu de sons. La Suède fut le pays qui, durant les années 1930, connut les liens les plus étroits avec les courants poétiques internationaux, notamment le surréalisme français, le mouvement littéraire de ce siècle qui a eu, sans doute, la plus grande influence sur la poésie du monde entier.

S'il fallait mentionner un poète Scandinave incarnant le modernisme même durant ces années, ce serait Gustav Munch-Petersen. Il est d'origine danno-suédoise. De nombreuses tendances convergent dans son œuvre. On y trouve une nette influence du groupe suédois Fem Unga (Cinq jeunes), ainsi que du modernisme finno-suédois, en particulier de Södergran et de Diktonius ; mais aussi celle de la poésie de la nature de Rabbe Enckell. Gustav Munch-Petersen était lié au groupe d'artistes et à la revue Linjen, et cette association avec la peinture reste typique. C'est elle qui aida à propager le surréalisme, tout autant que les études de présentation de Artur Lundkvist. Chez Munch-Petersen le radicalisme politique s'ajoute à un puissant investissement subjectif dans la langue, au rêve d'une nouvelle communauté fondée sur l'inconscient collectif, à un genre d'évangile primitiviste. Nous sommes dans la grande époque du freudianisme. Ses six recueils de poèmes sont une sorte d'enseignement de la conception moderniste du monde. Seul quatre d'entre eux furent imprimés de son vivant. Le premier porte un titre très expressionniste : Det nøgne menneske (L'Homme nu) (1932), le suivant rappelle la psychologie des profondeurs: Det underste land (Le Pays d'en-bas) (1933), puis vient le recueil surréaliste : Mod Jerusalem (Vers Jerusalem) (1934) puis enfin le recueil 19 digte (19 poèmes), écrit dans un style imagé anglo-saxon. Gustav Munch-Petersen prenait très au sérieux les grandes idéologies. Il s'engagea comme volontaire dans la guerre civile espagnole, pour lutter contre les fascistes, et mourut au front âgé seulement de 26 ans. Il laissa deux manuscrits qui furent publiés postérieurement : Solen finns (Le Soleil existe), écrit en suédois, avec des accents très nettement inspirés de Edith Södergran, et Black GoĎs Stone, écrit en langue anglaise, qui annonce le primitivisme des instincts, influencé par le romancier anglais D.H. Laurence, avec en arrière plan Walt Whitman.

algré toutes ces influences, la poésie de Munch-Petersen a un caractère très personnel. Elle ne fut absolument pas comprise à son époque, et les livres furent publiés à compte d'auteur. Les rares personnes à avoir fait des critiques de ses poèmes, pensaient qu'il avait l'esprit dérangé. Ce n'est qu'avec la vague du modernisme d'aprèsguerre qui déferla sur les pays nordiques - plus ou moins forte selon les régions - que l'époque fut mûre pour les comprendre. Depuis on lui dédie un culte.

On peut dire que la lutte avait été très dure avant que le modernisme dans son ensemble ne fût reconnu. La résistance était tenace aussi bien chez les lecteurs qu'au sein de l'institution littéraire. La polémique et les débats au sujet de l'incompréhensibilité de la poésie étaient encore à l'ordre du jour dans les années 1940 et 1950 , comme cela fût déjà le cas durant les premières années de l'avant-garde. Les poètes les plus connus furent les «Fyrtiotalistes " suédois (Poètes des années 40), qui créèrent dans cette Suède neutre durant la guerre une nouvelle poésie existentialiste, pleine de passions. Ce que l'on appelait alors « La catharsis de l'impuissance » en était un des thèmes caractéristiques - l'expérience de l'impuissance humaine et de son déchirement intérieur. C'était typiquement la tendance baudelairienne de la poésie. 
Son style chargé de métaphores y est reconnaissable dès les premières lignes de Mannen utan väg (L'Homme sans chemin) de Erik Lindegren (1942) :

Dans la salle des miroirs où non seulement Narcisse

trône sur le pilier de son désespoir, sans vertige,

l'éternité grimaçante suçait le lait

du pays des possibilités illimitées ${ }^{5}$.

Toute une série de représentations essentielles sont réunies ici : les miroirs, Narcisse, le désespoir, l'étourdissement, l'éternité, la grimace, les possibilités illimitées. Il est d'ailleurs caractéristique qu'aussi bien l'éternité que les possibilités illimitées apparaissent ici comme suspectes.

La poésie ne représente rien, elle ne décrit rien. Elle crée son propre monde, sa propre réalité linguistique. Elle affirme son autonomie. Elle peut menacer de se refermer sur elle-même et son propre processus de création - dans sa révolte contre le monde. Elle formule rarement un message, une fois que les manifestes ont fait leur temps. La poésie ne veut pas servir le pouvoir, elle est anarchiste. C'est sans doute là un trait essentiel du modernisme récent, bien que dans la phase de sa percée, il ait eu tendance à faire connaître publiquement ses idées, même dans une forme directe. La poésie est devenue une forme de catharsis, une purification, une constante expérimentation avec la langue et cela à une époque où la propagande politique avait tendance à dévoyer le langage. C'est pourquoi le modernisme risque en permanence d'être accusé de chercher l'esquive. Le modernisme peut être un exil: les poètes s'affirment dans une communication indirecte, en se posant contre leur époque.

51 Dans le monde occidental, dans le Nord aussi, la phase de la lutte du modernisme pour s'imposer est achevée. Il est accepté par tout le monde, ce qui représente un nouveau risque. Il est devenu déjà tradition, mais une tradition qui se renouvelle par intermittence. Précisément, car parfois le réalisme, cette poésie à caractère social, vient le remplacer. On peut même dire qu'il suit le calendrier. Après le modernisme intense des années 1940 vint une période de décompression dans les années 1950. Puis avec une nouvelle génération, le modernisme vécut un renouveau dans les années 1960, nettement influencé par la nouvelle philosophie du langage, par le structuralisme et la sémiotique. Ce fut ce que l'on appela la poésie thématique-scripturale qui inclut la langue, en tant que médium, dans sa réflexion. Au cours de cette décennie et au début des années 1970, la poésie est généralement caractérisée par une radicalisation politique et sociale, par le réalisme de la littérature féministe, une littérature dite engagée. Mais dans les années 1980, le modernisme est renouvelé un peu partout par une nouvelle génération, qui se sent en révolte contre les révoltes. Pourtant, il est clair que l'art moderne possède alors une longue tradition, riche et diverse, et que toute nouveauté est en même temps un retour, mis en système, à des styles antérieurs. On pourrait parler de modernisme stylistique ou de postmodernisme, où les modes d'expression historiquement déterminés sont abolis et où toutes les possibilités d'expression coexistent. C'est l'époque du mélange des styles.

J'ai commencé cette étude en appelant le modernisme une pieuvre. J'ai bien peur d'avoir donné effectivement cette impression, trop nettement même, avec une 
confusion de tentacules partant dans tous les sens et une eau de plus en plus trouble. Le concept de modernisme désigne-t-il, un contenu, une idéologie ou bien une période ?

On peut parler d'un monde de la modernité qui correspond à cette classe sociale que l'on appelle la bourgeoisie. Il commença au milieu du XVIII ${ }^{e}$ siècle en Europe et pris effectivement son essor au milieu du XIX ${ }^{e}$ siècle. Nous y rencontrons le modernisme « classique » de Withman et de Baudelaire, qui représentent respectivement le nouveau monde, avec la démocratie américaine, et l'ancien monde, avec la bourgeoisie du Second Empire en France. C'est là que furent jetées les bases du modernisme. Entre 1909 et 1924 nous eûmes le mouvement moderniste d'avant-garde qui, à travers un certain nombre de manifestations et de percées ultérieures a fini par créer une tradition reflétant une vision particulière de l'art: un art qui ne se réfère pas à la réalité mais qui s'y affronte, l'œuvre du poète justifiant par son écriture même sa propre réalité.

On pourrait dire aussi que le modernisme est une certaine manière de lire et de concevoir la littérature. Mais c'est là un autre point de vue, qui demanderait une toute autre étude. On pourrait penser en fin de compte, que le modernisme littéraire se limite simplement à la poésie. Ce n'est en aucune façon le cas. L'épopée et le drame, sans oublier les nombreuses formes mélangées et les transgressions des genres, sont une partie importante de son histoire. Considéré ainsi, la pieuvre possède au moins une dizaine de tentacules. Je me suis contenté ici de ne parler que d'une seule d'entre elles. Passablement longue déjà.

\section{NOTES}

1. « Jeg vet vad de leker

de leker ström, djuphavsström, frisk motström

som håller dem fladdrande på stället

över det lurande djupet ».

2. «Jeg seer paa den hvide himmel

jeg ser paa de graablaa skyer,

jeg ser paa den blodige sol.

Dette er altsaa verden.

Dette er altsaa klodernes hjem.

En regndraabe !

Jeg ser, jeg ser...

Jeg er vist kommet paa en feil klode !

Her er saa underligt... ».

3. «Stille! Se hvor Maskinen

den vældige Tingest, staar roligt og syder

og hykker sig i Røg, den er taalmodig. 
Tænd Piben paa fastende Liv,

forband Gud og svælg din Smærte ».

4. « Ångest, ångest är min arvedel,

min strupes sår,

mit hjärtes skri i världen.

Jeg skrattar med munnen full

av blod och vrålande hesa skri

åt dig ! ».

5. « i speglarnas sal där ej endast Narkissos

tronar på sin förtvivlans pelare utan svindel

diade evigheten med en grimas

de obegränsade möjligheternas Land».

\section{RÉSUMÉS}

Le modernisme, tel une pieuvre, est un animal étrange, insaisissable, en perpétuel mouvement. Ses multiples expressions prennent toujours leur point de départ dans un potentiel de protestation. À tout moment dans le modernisme on a le sentiment d'un abîme qui guette, d'une perte de sens, alors qu'en surface une danse endiablée traduit l'ivresse d'être délivré d'un vieux poids mort.

Les concepts admis généralement dans cette grande rupture avec les normes sont à la fois de nature positive et négative, la notion d'avant-garde restant, quant à elle, fondamentale. Le modernisme est inséparable de tout changement de civilisation. Deux voies d'accès, ouvertes l'une par Walt Whitman et l'autre par Charles Baudelaire, lui ont permis sa percée. Il est vrai qu'il s'est fait annoncer par tous les mouvements littéraires qui l'ont précédé, surtout en Scandinavie, naturalisme, symbolisme ou néo-romantisme. Les écrits de Johannes V. Jensen en fournissent une illustration typique.

La situation des -ismes en Europe au début du $\mathrm{xx}^{\mathrm{e}}$ siècle, cubisme, expressionnisme, dadaïsme, surréalisme avec son idée quasi dogmatique de l'ouverture à l'inconscient, montre que le plus souvent c'est la poésie, avec la peinture, qui est à l'origine du renouvellement. Face à cette situation, l'étude du modernisme nordique en littérature devient souvent un exercice de littérature comparée, un phénomène d'intertextualité. Edit Södergran ou Emil Bonnelycke et Tom Kristensen ? Finlande ou Danemark? Les historiens de la littérature sont divisés quant au lieu de naissance du modernisme nordique. Au Danemark l'année 1922, la plus importante dans l'histoire du modernisme, est exemplaire des scandales et polémiques qui accompagnent toujours ce mouvement.

En Suède, Pär Lagerkvist a été le premier à exprimer son extase devant l'homme nouveau et son angoisse existentielle. La première vague moderniste y eut lieu à la fin des années 20 , sous l'influence de Whitman, introduit par Artur Lundkvist; elle reste très marquée par le modernisme danois. En revanche, la deuxième vague ouverte vers 1940 par Erik Lindegren, y a connu des sommets et des réussites remarquables.

En Norvège, pays qui venait d'accéder à l'indépendance politique, le modernisme s'est plutôt manifesté en sourdine, en évitant les expérimentations formelles, malgré des auteurs de premier plan comme Knut Hamsun. S'il fallait mentionner un poète Scandinave incarnant le mieux le 
modernisme, ce serait probablement Gustav Munch-Petersen, dont l'œuvre est le point de convergence de nombreuses tendances. Publiée dans les années 30, elle ne fut absolument pas comprise. Ce n'est qu'avec la vague du modernisme des années d'après-guerre qu'on a commencé à lui dédier un véritable culte, reconnaissant que la poésie est anarchiste dans son essence même, une constante expérimentation avec la langue.

Dans le Nord, comme ailleurs, la phase de la lutte du modernisme pour s'imposer est achevée. Mais cela recèle un nouveau danger, car il risque déjà de devenir tradition à son tour. Alors, pour le renouveler encore, faut-il se sentir avec la génération des années 80 « en révolte contre les révoltes" ? Toute nouveauté est en même temps un retour. Il y a indéniablement un «monde de la modernité ", mais il possède de nombreuses tentacules. Le modernisme, cette " manière de lire et de dire ", n'est que Tune d'entre elles.

Der Modernismus ist ein gar seltsames Tier, eine Art Tintenschnecke, unfassbar und in ständiger Bewegung. Seine zahlreichen Ausdrucksformen gehen immer von einem gewissen Protestpotential aus. $\mathrm{Zu}$ jeder Zeit hat man beim Modernismus den Eindruck eines gähnenden Abgrundes, einer Desorientierung, während an der Oberfläche die trunkene Freude darüber, von einem alten toten Gewicht befreit zu sein, sich masslos auslässt.

Die allgemein bei diesem tiefen Bruch mit den hergebrachten Normen gültigen Konzepte sind zugleich positiver und negativer Art, während der Begriff der Avantgarde allenfalls seine fundamentale Bedeutung behält. Modernismus ist unzertrennlich von jeder Kultur Wandlung. Zwei verschiedene Ansätze, der eine durch Walt Whitman, der andere durch Charles Baudelaire eröffnet, haben ihm seinen Durch bruch ermöglicht. Er wurde zwar schon durch die literarischen Strömungen, die ihm vorausgingen, angekündigt, besonders in Skandinavien: Naturalismus, Symbolismus oder Neuromantik. Die Schriften von Johannes V. Jensen sind hier eine gute Illustrierung.

Die Gestaltung der -ismen in Europa zu Beginn des XX. Jahrhunderts, Kubismus, Expressionismus, Dadaismus, Surrealismus mit

seiner quasi dogmatischen Uberzeugung von der Notwendigkeit des Unbewussten, zeigt, dass meistens das Gedicht - und die Malerei - bei diesem Erneuerungsprozess ausschlaggebend sind. Angesichts dieser Lage erhält die Erforschung der Moderne im Norden eine stark komparativistische Prägung, im Lichte der Intertextualität.

Edith Södergran oder Emil Bonnelycke und Tom Kristensen? Finland oder Dänemark? Die Literaturhistoriker sind sich nicht einig über den Geburtsort der nordischen Moderne. Das Jahr 1922, das wichtigste übrigens in der Geschichte des Modernismus, ist in Dänemark exemplarisch für die Skandale und die Zänkereien, welche immer im Fahrwasser dieser Bewegung auftauchen.

Pär Lagerkvist ist der erste in Schweden gewesen, der seinem ekstatischen Rausch vor dem neuen Menschen und seiner existentiellen Angst Ausdruck verliehen hat. Die erste modernistische Welle erschien dort gegen Ende der 20er Jahre, unter dem Einfluss von Whitman, den Artur Lundkvist dort eingeführt hatte ; sie ist noch sehr stark vom dänischen Modernismus geprägt. Die zweite Welle aber, die sich gegen 1940, von Erik Lindegren angeregt, breit machte, hat literarische Höhepunkte und bemerkenswerte Erfolge gezeitigt.

In Norwegen, einem Land das soeben seine politische Unabhängigkeit erobert hatte, ist der Modernismus in gedämpfter Form in Erscheinung getreten, jede Experimentierung mit der Form wurde meistens vermieden, trotzdem das Land Verfasser ersten Ranges, wie Knut Hamsun, aufweisen konnte. Wäre ein einziger skandinavischer Dichter zu nennen, der am besten den Modernismus verkörpern würde, so wäre es wahrscheinlich Gustav Munch-Petersen, dessen Werk am Zusammenfluss vieler Strömungen steht. Es wurde in den Jahren 1930 veröffentlicht, aber absolut nicht verstanden. Nur mit der modernistischen Nachkriegswelle begann man, es anbeterisch ins Licht zu heben, in der Erkenntnis dass Poesie immer, ihrem Wesen nach, anarchistisch ist, eine stetige Experimentierung mit der Sprache. 
Im Norden, wie sonstwo in der Welt, ist die Phase, wo der Modernismus um seine Anerkennung ringen musste, beendigt. Gerade darin aber liegt eine neue Gefahr, denn er könnte schon selber wieder zur Tradition werden. Sollte man sich also, um ihn weiter zu erneuern, mit der Generation der Jahre 80 «im Aufruhr gegen jedweden Aufruhr » fühlen? Jede Neuerung ist zugleich eine Rückkehr. Es gibt wohl unleugbar eine "Welt der Modernität», aber sie hat vielfältige Fangarme. Der Modernismus, diese «Art zu lesen und zu sagen », ist nur einer unter den vielen.

\section{AUTEURS}

\section{TORBEN BROSTRøM}

Danmarks Laererhøjskole, Copenhague 